\title{
GESTIÓN DE LA SEGURIDAD Y SALUD DE LOS TRABAJADORES EN PYMES DE SERVICIOS EDITORIALES
}

\section{MANAGEMENT OF THE SAFETY AND HEALTH OF WORKERS IN PYMES OF EDITORIAL SERVICES}

\author{
Riera-Estrada Joe ${ }^{1}$; García-Moreira Sergio ${ }^{2}$; Chiriboga-Mendoza Mercedes ${ }^{3}$ \\ ${ }^{1}$ Profesor de la Universidad Laica Eloy Alfaro de Manabí, ULEAM. Manta, Ecuador. \\ ${ }^{2}$ Ingeniero Químico de la Universidad Técnica de Manabí, UTM. Portoviejo, Ecuador. \\ ${ }^{3}$ Médico familiar de la Universidad Católica del Ecuador, PUCE. Quito, Ecuador.
}

*Correo: sergamo1994@hotmail.com

\begin{abstract}
Resumen
Las empresas que buscan un crecimiento sostenible deben apuntar hacia estrategias que le otorguen ventajas competitivas frente a potenciales competidores del mismo sector. En las pequeñas y medianas empresas se genera la quinta parte de los accidentes laborales, por lo tanto, debe regularizarse un mayor control por parte de las instituciones públicas que cumplen estas funciones. Este trabajo pretende evaluar el contexto de una empresa de servicios editoriales que cuenta con 14 trabajadores para determinar la percepción de los colaboradores respecto a los accidentes laborales, riesgos y el grado de conocimiento y compromiso que se demuestra desde la alta directiva. La investigación se realizó mediante encuestas aplicadas al $100 \%$ de la población. Se determinó que el $79 \%$ de los encuestados considera que se presentan riesgos como caídas al mismo nivel, lo cual se relaciona con desorden por acumulación de cajas y material acumulado. Por otra parte, el $65 \%$ considera importante y necesaria la implantación de un sistema para la gestión de la seguridad y salud en el trabajo. Por lo tanto, la implementación de un sistema de gestión de seguridad y salud en el trabajo según la norma ISO 45001: 2018 permitirá organizar y planificar las actividades relacionadas con la prevención de riesgos laborales, así como la información documentada y la creación de procedimientos.
\end{abstract}

Palabras clave: riesgos, trabajo, normativa, planificación, ISO.

\begin{abstract}
Companies seeking sustainable growth should point to strategies that give them competitive advantages over potential competitors in the same sector. In small and medium enterprises, a fifth of work accidents are generated, therefore, greater control must be regularized by public institutions that fulfill these functions. This work aims to evaluate the context of a publishing services company that has 14 employees to determine the employees' perception regarding occupational accidents, risks and the degree of knowledge and commitment that is demonstrated by the senior management. The research was carried out through surveys applied to $100 \%$ of the population. It was determined that $79 \%$ of the respondents considered that risks arise as falls at the same level, which is related to disorder due to accumulation of boxes and accumulated material. On the other hand, $65 \%$ consider the implementation of a system for occupational safety and health management important and necessary. Therefore, the implementation of an occupational health and safety management system according to ISO 45001: 2018 will allow the organization and planning of activities related to occupational risk prevention, as well as documented information and the creation of procedures.
\end{abstract}

Keywords: risks, work, regulations, planning, ISO.

Información del manuscrito:

Fecha de recepción: 02 de agosto de 2019

Fecha de aceptación: 27 de noviembre de 2019

Fecha de publicación: 10 de enero de 2020 


\section{Introducción}

En el contexto actual es imposible minimizar el rol de la gestión en empresas, industrias y organizaciones, puesto que la alta competitividad y los avances continuos en materia de procesos y manejo administrativo exigen la aplicación de sistemas de gestión basados en procedimientos de mejora continua y enfocados en la excelencia. Aspectos como la calidad, el medio ambiente y la seguridad y salud en el trabajo son los principales focos de atención en la gestión de las organizaciones.

Las normas ISO proponen un sistema estándar para la gestión enfocada en el contexto situacional de la organización, planificación, medición, seguimiento, evaluación y mejora continua en lo que a nivel genérico se conoce como ciclo de Deming (PHVA). La norma ISO para la gestión de la seguridad y salud en el trabajo se denomina ISO 45001: 2018, resaltando que se ha constituido en un aspecto fundamental en la planificación laboral (ISO, 2018)., debido a que no solamente busca disminuir o evitar los accidentes laborales, sino también generar confianza y mayor desempeño laboral (Barrios \& Paravic, 2006).

El sector de la construcción es el más riesgoso en términos de accidentes laborales, asociándolo a accidentes como caídas, cortes, quemaduras; sin embargo, el sector de servicios ha adquirido relevancia en este análisis por la existencia de factores de riesgo relacionados con la organización, diseño de puestos de trabajo, sobrecarga y estrés, limpieza y falta de capacitación (Benavides et al., 2007). Es por esta razón, que se considera pertinente analizar la importancia y factibilidad de implantar la norma ISO 45001: 2018 en pequeñas y medianas empresas (pymes) del sector de servicios. Cabe señalar que según estadísticas propuestas por Acevedo \& Yánez (2016), el 18\% de los accidentes laborales se genera en organizaciones que no superan los 10 trabajadores, por lo tanto, son las pymes el principal punto de atención.

El objetivo del presente trabajo consiste en evaluar el contexto situacional de una organización clasificada como pyme y cuya actividad principal es la edición de textos. Además, se pretende determinar la necesidad y el impacto 
que generaría la implantación de la norma ISO 45001: 2018, para la gestión de la seguridad y salud de los trabajadores.

\section{Materiales y métodos}

Se define a la investigación como descriptiva y con un enfoque cuantitativo. Se aplicaron encuestas a los 14 trabajadores de una empresa dedicada a la edición y diseño de textos académicos y científicos en la ciudad de Portoviejo, Manabí, Ecuador. El trabajo de campo para recolectar la información se realizó entre junio y julio del 2019.

Se aplicó un instrumento de investigación que cuenta con información del aportante: edad, sexo, tiempo de labores y jornada de trabajo; la encuesta contiene 10 preguntas cerradas que permitieron determinar la percepción de los trabajadores respecto al manejo administrativo y gestión de la seguridad y salud, casos de riesgos laborales, factores de riesgos laborales y estrategias de la empresa para precautelar la integridad física y psicológica de los trabajadores.

\subsection{Muestra}

Se realizaron encuestas físicas al $100 \%$ de la población de trabajadores de la organización, puesto que se trata de una pyme que cuenta con 14 trabajadores.

\subsection{Participantes}

La muestra analizada estuvo compuesta por 14 trabajadores, de los cuales 6 son hombres y 8 son mujeres. Respecto a la edad, las mujeres tienen una edad que oscila entre 32 y 46 años, por su parte los hombres entre 29 y 48 años.

A partir de la información obtenida en la investigación se sistematizó la información del contexto de la institución en una matriz FODA (fortalezas, oportunidades, debilidades y amenazas). Además, se revisó la bibliografía referente para discutir los resultados obtenidos en la presente investigación con manuscritos propuestos para otras organizaciones.

\section{Resultados y discusión}

La organización analizada es una institución que se dedica a la edición y producción de libros y que, si bien no ha sufrido graves consecuencias en términos de accidentes laborales, 
se ha detectado la presencia de factores de riesgo que pueden ocasionar accidentes, incidentes y no conformidades.

Tabla 1.

Análisis del contexto situacional de la pyme de servicios editoriales

\begin{tabular}{|c|c|}
\hline Fortalezas & Debilidades \\
\hline $\begin{array}{l}\text { - La empresa cuenta con varios } \\
\text { años en el mercado y tiene un } \\
\text { segmento del mercado } \\
\text { asegurado; además, cuenta con } \\
\text { marca difundida en el medio. } \\
\text { - La organización cuenta con el } \\
\text { 100\% de los trabajadores afiliados } \\
\text { al Instituto Ecuatoriano de } \\
\text { Seguridad Social. } \\
\text { - La gerencia de la empresa está } \\
\text { interesada y comprometida con la } \\
\text { implementación de las normas } \\
\text { ISO, especialmente con la norma } \\
45001: 2018 \text {. }\end{array}$ & $\begin{array}{l}\text { - Gestión nula de la seguridad y } \\
\text { salud en el trabajo. } \\
\text { - Espacios de trabajo y condiciones } \\
\text { laborales que no cumplen con los } \\
\text { requisitos mínimos para garantizar } \\
\text { la seguridad y salud. } \\
\text { - No se documenta toda la } \\
\text { información generada en el } \\
\text { seguimiento de los procesos y } \\
\text { puntos críticos; ni tampoco se } \\
\text { gestionan auditorías de revisión. }\end{array}$ \\
\hline Oportunidades & Amenazas \\
\hline 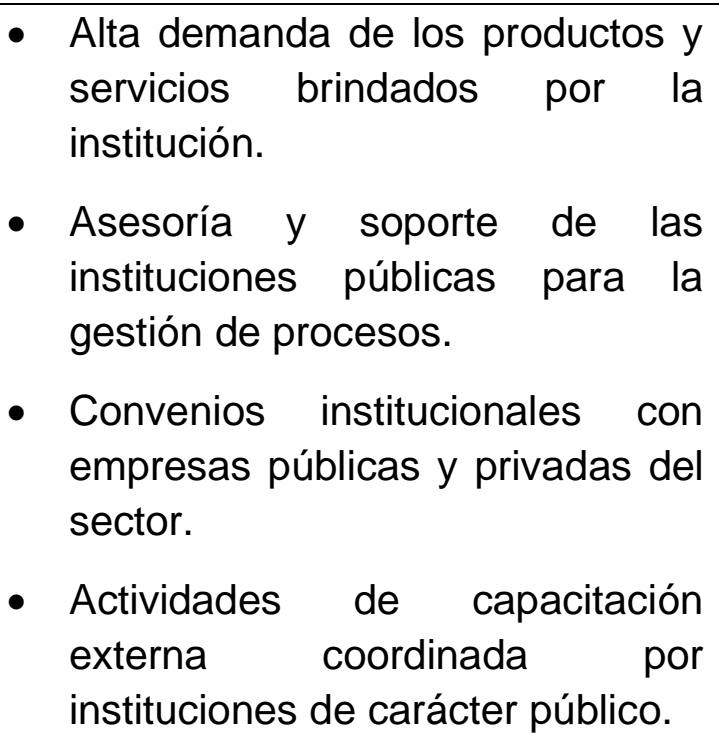 & 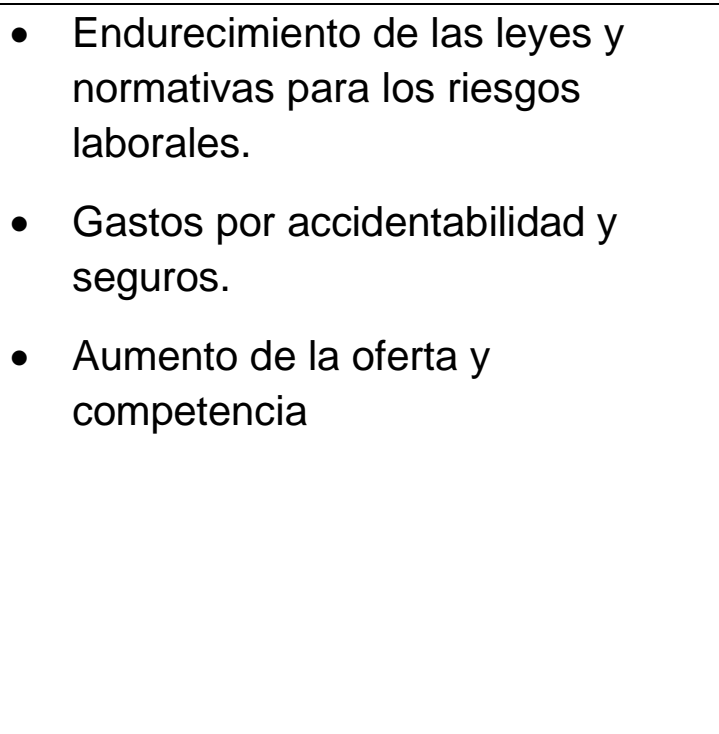 \\
\hline
\end{tabular}

Fuente: Elaboración propia.

En la tabla 1 se muestran los resultados del análisis situacional generado a partir de las encuestas realizadas a los colaboradores de la organización. Se determinó que si bien no se han presentado 
accidentes que atenten contra la vida de los trabajadores, existen varios factores de riesgo que implican que en cualquier momento se generen accidentes e incidentes y no conformidades. El $79 \%$ de los encuestados considera que se presentan riesgos como caídas al mismo nivel, lo cual se debe en que ciertos puntos se acumulan objetos y materiales que no se usan periódicamente. Asimismo, se indica que un $38 \%$ ha sufrido cortes con materiales de trabajo y no se han presentado ni quemaduras ni lesiones mayores, a pesar de que existen factores de riesgo que se encuentran latentes. Existen empresas que han demostrado un aumento en la eficiencia de sus procesos, tras la aplicación de políticas que permitieron sustituir actividades de producción con riesgo físico con actividades de prestación de servicios (Zhang, 2009).

Por otra parte, el $65 \%$ señala que durante los días finales del mes les toca realizar actividades extra, generando estrés y sobrecarga mental.

Cabe indicar que los trabajadores consideran que la gerencia tiene muy pocos conocimientos acerca de la gestión de riesgos laborales y que es fundamental que se instruyan y planifiquen capacitaciones para todos los miembros de la organización. En este caso, el 93\% de los trabajadores afirma que es fundamental desarrollar una cultura de prevención de riesgos laborales (PRL) e implantar un sistema de gestión de la seguridad y salud de los trabajadores. Cortés et al., (2014) determinaron que el $70,06 \%$ de la población encuestada en el estudio indica que la prevención de riesgos genera aspectos positivos en la organización, tales como prestigio, posicionamiento, relación con clientes y trabajadores, mejora continua de procesos y procedimientos. Además, un $41 \%$ de los participantes en dicho estudio indican que la prevención de riesgos genera mayor productividad en los trabajadores. No obstante, en la presente investigación se concluye que el $65 \%$ de los participantes señala que la adopción de medidas de PRL y la implantación de un sistema de gestión de seguridad y salud en el trabajo promueven un mayor compromiso en los colaboradores, puesto que se sienten respaldados bajo condiciones laborales óptimas para 
rendir al máximo en sus funciones (Edvinsson \& Malone, 2000).

Castilla (2003) señala que las pymes consideran a los recursos intangibles como una apuesta para adquirir competitividad en el mercado. Es válido señalar que los accidentes laborales generan un deterioro en los recursos de la empresa, comprometiendo así la sostenibilidad de la empresa en el mediano y largo plazo (Fernández et al., 2006).

Las dos aristas de las potenciales consecuencias de los accidentes de trabajo se fundamentan en la generación de costos financieros y de oportunidad (Gil \& Pujol, 2000) y en la caída de la calidad y cantidad de producción.

Desde al análisis realizado, se plantea como un mecanismo para optimizar la gestión de seguridad en el trabajo, la implantación del sistema de gestión de seguridad y salud de los trabajadores basado en la norma ISO 45001: 2018. Los primeros requisitos que se proponen son:

- Elaboración de una política para la gestión de seguridad y salud.
- Elaboración del mapa de procesos.

- Desarrollo de un análisis de la situación actual de la organización mediante la matriz FODA.

- Identificación de partes involucradas en el sistema.

- Determinación de peligros y la aplicación de un método para la evaluación de riesgos.

La norma exige una serie de requisitos mínimos para la implantación del sistema de gestión, sin embargo, cada organización puede aplicar las estrategias, metodologías y procedimientos que considere pertinentes. El manual del sistema de gestión de seguridad y salud para las pymes de servicios editoriales debe recoger los mecanismos para la organización sigue para cumplir con los requisitos, definiendo responsables y los tiempos requeridos para ejecutar el criterio. Por tanto, la empresa implanta este sistema de gestión con la misiva de reducir la siniestralidad y a la vez generar ventajas competitivas que le permitan optimizar la rentabilidad, mejorar la productividad y crear intangibles a 
favor de la organización (Sorensen, 2002; García-Miranda et al., 2010).

\section{Conclusiones}

La seguridad y salud de los trabajadores ya no es un requisito que deben cumplir las organizaciones para disminuir 0 evitar los accidentes laborales; sino más bien se define como una estrategia empresarial para generar mayor confianza entre proveedores, clientes y trabajadores. Además, permite evitar potenciales costos por accidentes laborales e interrupciones en las actividades desarrolladas por la institución, generando de esta forma ventajas tangibles e intangibles.

Condiciones de trabajo inseguras e insalubres bajan la moral de los colaboradores y disminuyen su desempeño, generando incluso renuncias al trabajo. Por lo tanto, la implementación de un sistema de gestión de seguridad y salud en el trabajo según la norma ISO 45001: 2018 permitirá organizar y planificar las actividades relacionadas con la prevención de riesgos laborales, así como la información documentada y la creación de procedimientos para reportar todo de tipo de accidentes, incidentes y no conformidades, garantizando los procesos de mejora continua.

\section{Bibliografía}

Acevedo, K. \& Yánez, M. (2016). Costos de los accidentes laborales: CartagenaColombia, 2009-2012. Ciencias Psicológicas, 10(1), 31-41.

Barrios-Casas, S. B., \& Paravic-Klijn, T. P. (2006). Promoción de la salud y su entorno laboral saludable. Rev Latino-am Enfermagem, 14(1), 136-41.

Benavides, F., Amable, M., Boix, P., Castejón, E., Bosch, C., García, A., Gil, J. M., Moncada-Lluís, S., Jódar, P., \& Rodrigo, F. (2007). Informe de Salud Laboral. España, 2006-2007. Obtenido de: http://hdl.handle.net/2072/410 3

Castilla, F. (2003). La dimensión inmaterial como apuesta competitiva de PYMES. Revista de Contabilidad y Tributación CEF, 248: 248249.

Edvinsson, L. \& Malone, M. (2000). El capital intelectual. Cómo identificar y calcular el valor de los recursos intangibles de su empresa. Barcelona: Gestión 2000.

Fernández, B., Montes, J. \& Vázquez, C. (2006). La gestión de la seguridad laboral: incidencia sobre los 
resultados de la organización. Revista Europea de Dirección y Economía de la Empresa, 16(1): 115-136.

García-Miranda, C. E., Muñiz, B. F., \& Sánchez, A. S. (2010). Organización de la actividad preventiva y gestión de la seguridad y salud laboral en la minería española: experiencia de las empresas certificadas ISO 9001. Dirección y Organización, (40), 86-98.

Gil, A., \& Pujol, L. (2000). Metodología para la evaluación económica de los accidentes de trabajo. Prevención, trabajo y salud, 7 , 27-39.

ISO. (2018). Sistema de gestión de seguridad y salud en el trabajo. International Organization for
Standardization: ISO 45001: 2018.

Sorensen, J. N. (2002). Safety culture: a survey of the stateof-the-art.

Reliability Engineering \& System Safety, 76(2), 189-204. https://doi.org/10.1016/S0951 -8320(02)00005-4

Zhang, X. (2009). Producer service and the added value of manufacturing industries. An empirical research based on various industries of different countries. International Journal of Economics and Finance, 1(2), 21-26. 